\title{
Pedagogía Universitaria Basada en Competencias Genéricas para Desarrollar Habilidades del Pensamiento Crítico en Estudiantes de la Universidad Nacional de San Martín
}

\section{University Pedagogy Based on Generic Competencies to Develop Critical Thinking Skills in Students of the National University of San Martín}

\author{
Ibis López-Novoa* \\ Universidad Nacional de San Martin, Tarapoto, Perú \\ ORCID: https://orcid.org/0000-0001-6387-3376 \\ Manuel Padilla-Guzmán \\ Universidad Nacional de San Martin, Tarapoto, Perú \\ ORCID: https://orcid.org/0000-0002-1799-1221 \\ Mónica Juárez-De La Cruz \\ Universidad Nacional de San Martin, Tarapoto, Perú \\ ORCID: https://orcid.org/0000-0001-8733-4369 \\ Santiago Gallarday-Morales \\ Universidad Nacional de Educación Enrique Guzmán y Valle, Lima, Perú \\ ORCID: https://orcid.org/0000-0002-0452-5862 \\ Yrene Cecilia Uribe Hernández \\ Universidad Nacional de Cañete, Cañete, Perú \\ ORCID: https://orcid.org/0000-0001-5893-9262
}

Recibido 12-12-19 Revisado 13-02-20 Aprobado 28-06-20 En línea 04-07-20

Correspondencia

Email: illopeznovoa@unsm.edu.pe
Citar como:
López-Novoa, I., Padilla-Guzmán, M., Juárez-De La Cruz, M., Gallarday Morales, S., Uribe, Y. (2020). Pedagogía Universitaria Basada en Competencias Genéricas para Desarrollar Habilidades del Pensamiento Crítico en Estudiantes de la Universidad Nacional de San Martín. Propósitos y Representaciones, 8(3). doi: http://dx.doi.org/10.20511/pyr2020.v8n3.561 


\section{Resmen}

El presente artículo aborda el tema de la pedagogía universitaria basada en competencias genéricas para desarrollar las habilidades del pensamiento crítico, el objetivo fue Medir la influencia de la pedagogía universitaria basada en competencias genéricas en el desarrollo de habilidades del pensamiento crítico en los estudiantes del I ciclo de la Universidad Nacional de San Martín, 2019. La investigación fue cuantitativa, diseño cuasi experimental, de tipo experimental, la población lo conformaron 420 estudiantes, siendo la muestra determinada por 180 estudiantes, a quienes se les aplico un cuestionario a modo de encuesta para recoger la información. Los resultados muestran

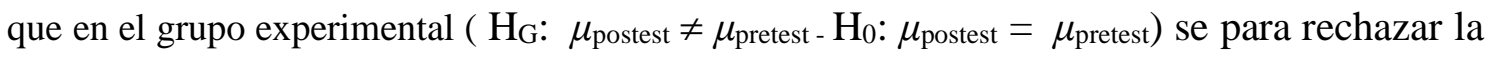
hipótesis nula, por tanto los puntajes son diferentes, los puntajes son mayores en el pos test comparado con el pre test $(\mathrm{P}$ sig $<0,05-0,000<0,05)$ en este caso se rechaza la hipótesis nula y se acepta la hipótesis alternativa donde la efectividad de la pedagogía universitaria basada en competencias genéricas en el desarrollo de habilidades del pensamiento crítico en los estudiantes del I ciclo de la Universidad Nacional de San Martín, 2019; es decir los puntajes del pos test son altos

Palabras Clave: Pedagogía universitaria; competencias genéricas; pensamiento crítico; inferencia; explicación; autorregulación.

\section{Sumary}

This article addresses the issue of university pedagogy based on generic skills to develop critical thinking skills, the objective was to measure the influence of university pedagogy based on generic skills in the development of critical thinking skills in students in the I cycle from the National University of San Martín, 2019. The research was quantitative, quasi-experimental design, experimental type, the population was made up of 420 students, the sample determined by 180 students, to whom a questionnaire was applied as a survey to Collect the information. The results show that in the experimental group $\left(\mathrm{H}_{\mathrm{G}}: \mu_{\text {postest }} \neq \mu_{\text {pretest }}-\mathrm{H}_{0}: \mu_{\text {postest }}=\mu_{\text {pretest }}\right)$ the null hypothesis is rejected, therefore the scores are different, the scores are higher in the post test compared to the pre test $(\mathrm{P}$ sig $<0.05-0.000<0.05)$ in this case the null hypothesis is rejected and the alternative hypothesis is accepted where the effectiveness of university pedagogy based on generic competencies in the development of critical thinking skills in I students cycle of the National University of San Martín, 2019; that is the post test scores are high.

Palabras Clave: University pedagogy; generic skills; critical thinking; inference; Explanation; self-regulation..

\section{Introducción}

El modelo educativo universitario es una representación de la realidad, en este caso de los principales sistemas y subsistemas que intervienen en el proceso de formación de profesionales en las universidades. La necesidad de definir un modelo educativo en la universidad es tal, que constituye un indicador importante para determinar la calidad educativa.

Durante los primeros años del milenio actual, las reflexiones sobre la universidad del futuro ocuparon un lugar preponderante en el escenario educativo mundial y plantearon retos importantes para el desarrollo de las universidades del futuro, entre los cuales destaca la definición de modelos educativos acordes a las realidades cambiantes. Es debido a lo anterior, que nuestra 
universidad se propuso seguir un modelo de formación humanista, basado en la construcción de conocimientos y centrado en el aprendizaje que muestre además un enfoque tanto multidisciplinario como interdisciplinario, esquemas operativos flexibles y compromiso social.

En el contexto actual de nuestro país, resulta importante, e incluso prioritario, formar profesionistas conscientes, críticos y sensibles a los diversos problemas que enfrenta la sociedad actual: sus desigualdades y sus procesos de crecimiento y cambio (Hernández et ál., 2015, como se citó en Pineda et ál., 2017)

Consecuentemente, los desafíos que la educación superior universitaria debe asumir son cuatro aspectos para la vida universitaria: a) reconocer la importancia de la investigación para el desarrollo sustentable y fomentarla debidamente; b) la urgente búsqueda de excelencia y calidad en todas las actividades que las universidades realizan; c) la ineludible responsabilidad de los Estados en la educación superior como bien público; y d) la urgencia de ofrecer un mejor trato a los docentes universitarios. Y ¿cómo asumiremos estos desafíos? Haciendo que, en la universidad, se cumpla con la legislación universitaria instituidos en los principios, fines y funciones de la universidad establecidos en la nueva ley universitaria 30220 y entrar a un proceso de actualización científica e intervenir en la innovación tecnológica. (Minedu, 2014).

\section{Pedagogía universitaria.}

La pedagogía universitaria busca que los estudiantes desarrollen una comprensión profunda de su entorno, que desarrollen una consciencia crítica de su rol como ciudadanos, así como la disposición y preparación para comprometerse una la construcción de una sociedad más justa y equitativa. Son propuestas pedagógicas orientadas a asegurar que los docentes logren que los estudiantes construyan conocimientos profundos, significativos, relevante, que les permitan insertarse adecuadamente en el mundo académico y profesional. Aquí encontramos a autores como Biggs (2011), Bain (2004), y un conjunto de académicos e investigadores que provienen de la corriente que busca el desarrollo y la profesionalización de la enseñanza en el nivel superior.

Según Kemmis (2012) retoma la noción de pedagogía como praxis en el sentido filosófico estoico y propone una pedagogía universitaria estrechamente vinculada a la praxis profesional y académica situada social y políticamente. Barnett (1999) propone renovar la pedagogía universitaria de forma tal que prepare a los estudiantes para afronta la "supercomplejidad" del mundo actual, en el cual nociones fundamentales como verdad y justicia muchas tienen interpretaciones contenciosas entre las cuáles es difícil tomar partido.

La pedagógica del docente universitario considerando el concepto de educación y sus fines, las implicancias éticas derivadas de una determinada conceptualización de la educación, los fines de la universidad, las tareas del trabajo universitario y la razón de ser docente, a los efectos de entender con claridad los enunciados del presente trabajo.

Según Polar (2010), manifestó que la educación consiste en el mejoramiento, en el perfeccionamiento intencional y en el crecimiento integral de la persona mediante la ayuda del educador; educar es personalizar; la persona da unidad al proceso educativo. Para todo docente universitario, que pretenda ser docente educador, hay exigencias necesarias sin las cuales no habrá educación eficaz. 


\section{Didáctica universitaria}

Álvarez de Zayas (1998), pionero en establecer una didáctica para la educación superior, basada en su teoría de los procesos conscientes, que a su vez, se fundamenta en la teoría de la concepción de los procesos sociales de González (1993) y con base filosófica en el materialismo dialéctico e histórico.

Partiendo de este mismo marco teórico, se han elaborado otros modelos de didáctica para la educación superior; Salazar (2001). Estos modelos han enriquecido la epistemología de la didáctica de la educación superior y han aparecido definiciones de nuevas categorías didácticas.

La didáctica universitaria se basa en las prácticas. Es a través del estudio de la práctica, del contraste de los resultados obtenidos, del análisis de las concepciones y supuestos desde los que se ha llevado a cabo, etc. como se va construyendo el conocimiento didáctico. Eso supone que la mejor manera de formarse como docente (sin olvidar esos conocimientos generales y de fundamentación que servirán de base previa al ejercicio de la docencia) es el análisis y revisión de la práctica real. No es que uno haya de aprenderlo todo de su propia práctica, como si fuera el único referente de conocimiento de que podrá hacer uso (Zabalza, 2011).

También existe conocimiento didáctico acumulado, como en cualquier otra disciplina. Pero es un conocimiento que se ha ido construyendo a partir de la práctica, de nuestra práctica y de la práctica de los demás, de la que se hace ahora y de la que se hizo y se estudió en otras épocas. Pero incluso así, no deja de repetirse, hoy en día, la importancia de revisar la propia práctica, de reflexionar sobre ella. En ello radica nuestra mejor garantía para ir mejorando nuestras competencias como docentes.

La Didáctica universitaria puede asumir de cara a la transformación profesional de los docentes y la mejora de la práctica formativa que desarrollan. Una de las condiciones fundamentales para la mejora de la calidad de la docencia universitaria radica, a mi humilde entender, en hacer posible que se produzca un cambio sustantivo en la configuración de la identidad profesional de los docentes universitarios.

Todos los profesores y profesoras universitarias son didactas, todos ellos y ellas enseñan y deben poseer las dotes (las competencias) que les permitan llevar a cabo esa tarea: el enseñar con eficacia; en ello radica uno de los puntos fuertes de nuestra identidad profesional. Seguramente también somos investigadores y algunos habremos asumido diferentes responsabilidades en la gestión de nuestras instituciones. Pero si hay algo que unifica nuestra función y nuestro desempeño profesional es que somos docentes

Al hablar de docencia universitaria, no es infrecuente escuchar a algunos colegas que dicen, "bueno, eso está bien a nivel general, pero en nuestra carrera las cosas son distintas". Efectivamente, puede que lo sean, que ellos lo estén haciendo de manera diversa (organizar las clases, evaluar, orientar a los estudiantes, etc.) pero no porque necesariamente hayan de hacerlo así sino porque ésa es la costumbre en su institución. O quizás porque no se han preocupado en formarse para poder hacerlo mejor. Como en tantas otras cosas de la vida, es mucho más lo que tiene de común la docencia de las diversas especialidades académicas que lo que tiene de distinto. Aunque, por supuesto, suele resultar más frecuente fijarse en lo distinto que hacerlo en lo común. Lo cual, al final, redunda en perjuicio de todos. Aprenderíamos mucho más si tuviéramos una visión holística de los procesos de enseñanza aprendizaje. 


\section{Competencias genéricas}

Las competencias genéricas son habilidades o destrezas, actitudes y conocimientos transversales que se requieren en cualquier área profesional, que son transferibles a una gran variedad de ámbitos de desempeño y que fortalecen la empleabilidad. Estas competencias son potenciadas principalmente a través de metodologías activas centradas en el estudiante y en su desarrollo interactúan elementos de orden cognitivo y motivacional. Son aquellas competencias que apuntan a la movilización de recursos personales (conocimientos, habilidades y actitudes) y recursos del ambiente, con relación a fines considerados importantes para todo desempeño, independientemente de la función o nivel.

La competencia genérica propiamente tal, se define como "un sistema complejo de conocimientos, integrados por las dimensiones cognitiva, afectiva y procedimental, observables en el desempeño, y que pueden ser implementadas en distintos contextos, es sensible al arte y participa en la apreciación e interpretación de sus expresiones en distintos géneros (Navarro, 2015).

En cuanto a competencia genérica, se identifica para este estudio, como aquella que es común a varias ocupaciones o profesiones, lo que para el presente caso refiere al desempeño de todo docente en la Universidad. Formar este tipo de competencia, permite afrontar los continuos cambios propios del quehacer profesional; aumenta las posibilidades de adquisición de empleo; favorece la gestión y conservación del empleo y la adaptación a diversos ambientes laborales; además, adquirir una competencia de este tipo, apela a un proceso sistemático de aprendizaje, y su desempeño, a una evaluación rigurosa (Tobón, 2005).

\section{Habilidades del pensamiento crítico}

Para Péres y Gardey (2011) La habilidad es la capacidad y disposición para algo. El concepto puede usarse para nombrar al grado de competencia de un sujeto frente a un objetivo. Es importante destacar que la habilidad puede ser innata o desarrollada a partir del entrenamiento, la práctica y la experiencia).

Guzmán y Sánchez (2006), señalan que el pensamiento crítico se ha relacionado, frecuentemente, con el uso de herramientas cognitivas que permiten aumentar la posibilidad de alcanzar un cierto resultado deseable. Se le ha descrito como un proceso intencionado de pensamiento que está orientado al logro de una meta, como el tipo de pensamiento que se utiliza en la resolución de problemas, la toma de decisiones, el análisis y las inferencias lógicas. De alguna manera, todas estas definiciones implican procesos mentales que son útiles para una tarea cognitiva particular; implica un pensamiento con una dirección ya que se enfoca en la obtención de un resultado deseado.

Laskey y Gibson (1997) afirman que el pensamiento crítico es un proceso complejo que hace referencia a un repertorio de actividades cognitivas que actúan de manera conjunta, y que incluyen habilidades cognitivas, tales como: resolución de problemas, pensamiento lógico, perspectiva y percepción de ideas; análisis, evaluación y toma de decisiones. Estos autores apoyan la idea de que los profesores deberían usar preguntas dirigidas, con el fin de desarrollar el pensamiento crítico en sus estudiantes universitarios.

El pensamiento crítico, en cambio, supone otro tipo de habilidades, como ser: el juicio, la crítica y la opinión: nos permiten realizar el análisis de los datos que percibimos, para más tarde usarlos como base en la concepción que tenemos de nuestro entorno; la evaluación: sirve para la 
emisión de juicios de valor que, a su vez, nos conducen a decidir qué camino tomar a cada paso; la metacognición: nos hace conscientes de nuestro propio accionar y de nuestros procesos mentales.

El pensamiento crítico es una habilidad que todo ser humano debe desarrollar ya que tiene cualidades muy específicas y que nos ayudan a resolver problemas de una mejor manera, nos hace más analíticos, nos ayuda a saber clasificar la información en viable y no viable, nos hace más curiosos, querer saber e investigar más acerca de temas de interés. Cuando se desarrollan este tipo de habilidades, también se desarrollan muchas otras capacidades del cerebro como la creatividad, la intuición, la razón y la lógica, entre otras. (Paul y Elder, 2005).

Para Brookfield (1987) argumenta que las habilidades de pensamiento crítico son vitales para llegar a ser una persona plenamente desarrollada. A pesar de que muchos educadores reconocen la necesidad de ayudar a sus estudiantes a desarrollar estas destrezas, muchos profesores sienten que no tiene suficiente tiempo para dedicarlo a esta meta, algunos otros han reconocido su falta de habilidad para pensar de manera crítica y, por tanto, no se sienten aptos para enfrentar este reto. El autor ha identificado cuatro características de los pensadores críticos: a) Tratan de identificar los supuestos que subyacen las ideas, las creencias, los valores y las acciones; b) están conscientes del contexto; c) poseen la capacidad de imaginar y explorar alternativas a maneras existentes de pensar y de vivir; d) usualmente son escépticos a afirmaciones de verdades universales o explicaciones últimas y definitivas.

\section{Dimensiones del pensamiento crítico}

Las dimensiones del desarrollo de pensamiento crítico son: interpretación, análisis, inferencia, explicación y autorregulación. Si bien se trata de niveles superiores de pensamiento, su importancia en el área de la salud radica en la aplicación de su práctica, el material humano. (Olivares et al., 2016).

Según Giancarlo y Facione (2001) identificaron además seis habilidades centrales para el pensamiento crítico: Análisis, inferencia, interpretación, evaluación, explicación y autorregulación, y llegaron al consenso de que el pensamiento crítico es un fenómeno humano intencional y persuasivo. Por lo tanto, las personas que piensan de manera crítica no sólo se caracterizan por sus destrezas cognitivas, sino además, por la manera como ven la vida; estas personas pueden ser reconocidas por cómo afrontan las preguntas, los asuntos o los problemas. Esto quiere decir que el pensamiento crítico va más allá del salón de clases.

En la taxonomía de Bloom (1990) independientemente de la metodología que utilicemos en nuestra práctica educativa Bloom clasifica las siguientes habilidades: Conocimiento, comprensión, aplicación, análisis, síntesis y evaluación.

\section{Pensamiento crítico inferencial.}

La inferencia es el proceso por el cual se derivan conclusiones a partir de premisas. Cuando una proposición se sigue de otras de ese modo, se dice que éstas implican aquella. Cuando una inferencia es aceptable, lo es por su estructura lógica y no por el contenido específico del argumento o el lenguaje utilizado. Por esto se construyen sistemas formales que capturan los factores relevantes de las deducciones como aparecen en el lenguaje natural. Tradicionalmente se 
distinguen tres clases de inferencias: las deducciones, las inducciones y las abducciones, aunque a veces se cuenta a la abducción como un caso especial de inducción (Encyclopedia Britannica, 2009).

El pensamiento crítico inferencial hace referencia al proceso de "identificar y asegurar los elementos necesarios para sacar conclusiones razonables; formular conjeturas e hipótesis; considerar la información pertinente y sacar las consecuencias que se desprendan de los datos, enunciados, principios, evidencia, juicios, creencias, opiniones, conceptos, descripciones, preguntas u otras formas de representación". Como sub habilidades de inferencia, los expertos incluyen cuestionar la evidencia, proponer alternativas, y sacar conclusiones. ¿Puede pensar en algunos ejemplos de inferencia? Quizás sugiera cosas como ver las implicaciones de la posición asumida por alguien, o extraer o construir significado de los elementos de una lectura.

Anticipando o construyendo quizás qué sucederá a continuación basado en lo que se conoce sobre las fuerzas en juego en una situación dada, o formulando una síntesis de ideas relacionadas en una perspectiva coherente. ¿Qué tal si después de determinar que para usted sería útil resolver cierta indecisión, desarrollara un plan de trabajo para recoger la información necesaria? ¿O, si al encontrar un problema, propusiera una serie de opciones para afrontarlo? ¿Qué tal conducir un experimento controlado científicamente y aplicar los métodos estadísticos adecuados para intentar confirmar o refutar una hipótesis empírica?

\section{Pensamiento crítico explicativo}

La explicación debe estar determinada como concepto, discurso, ley, génesis, causa, o descripción, de forma que siempre que se dieran las mismas condiciones se espera que se producirán los mismos efectos (en el terreno de los hechos, cuando se trata de sucesión hechos), o las mismas explicaciones (cuando se trate de comprensión de significados de conceptos o discursos), para cualquier observador humano en iguales circunstancias (Nagel, 2006).

Se considera al pensamiento crítico explicativo como la capacidad de presentar los resultados del razonamiento propio de manera reflexiva y coherente. Esto significa poder presentar a alguien una visión del panorama completo: "tanto para enunciar y justificar ese razonamiento en términos de las consideraciones de evidencia, conceptuales, metodológicas, de criterio y contextuales en las que se basaron los resultados obtenidos; como para presentar el razonamiento en forma de argumentos muy sólidos".

Las sub habilidades de la explicación son describir métodos y resultados, justificar procedimientos, proponer y defender, con buenas razones, las explicaciones propias causales y conceptuales de eventos o puntos de vista y presentar argumentos completos y bien razonados en el contexto de buscar la mayor comprensión posible. Ahora, sus ejemplos, por favor... Aquí hay algunos más: elaborar un cuadro que organice los hallazgos propios; escribir, para referencias futuras, su pensamiento actual respecto de algún asunto importante y complejo; citar los estándares y los factores contextuales utilizados para juzgar la calidad de una interpretación de un texto; enunciar los resultados de una investigación y describir los métodos y criterios utilizados para alcanzar dichos resultados; apelar a criterios establecidos para demostrar la sensatez de un juicio dado; diseñar una presentación gráfica que represente con precisión las relaciones de subordinación y de supraordinación entre conceptos o ideas; ubicar la evidencia que lo llevó a aceptar o a rechazar la posición de un autor con respecto a un asunto; listar los factores que se tuvieron en cuenta para asignar la calificación final a un curso.

Sin embargo, quizás la habilidad cognitiva más extraordinaria de todas, es esta última; es extraordinaria porque permite a los buenos pensadores críticos mejorar su propio pensamiento; 
en cierto sentido se trata del pensamiento crítico aplicado a sí mismo. Debido a esto, algunas personas quieren llamarlo "metacognición", que significa elevar el pensamiento a otro nivel. Pero ese "otro nivel" no lo capta totalmente, porque en ese siguiente nivel lo que hace la autorregulación es mirar en retrospectiva todas las dimensiones del pensamiento crítico y auto verificarse. La autorregulación es como una función recursiva en términos matemáticos, esto es que puede aplicarse a todo, incluso a ella misma. Usted puede revisar y corregir una interpretación que presentó. Puede examinar y corregir una inferencia que haya hecho. Puede revisar y reformular una de sus propias explicaciones. ¡Incluso puede examinar y corregir su habilidad para examinarse y auto corregirse! "¿cómo lo estoy haciendo? ¿he omitido algo importante? Voy a verificar antes de ir más allá”

\section{Pensamiento crítico autorregulado}

La autorregulación se refiere a la autogeneración de pensamientos, sentimientos y acciones, que las personas utilizamos para conseguir sus metas, en una visión triádica y recíproca. Cuando los estudiantes trabajan en sus tareas, regulan sus factores personales, estableciendo metas, monitoreando y autoevaluando sus progresos, y evaluando su autoeficacia para continuar aprendiendo y crear así un ambiente positivo para ellos. Desde una visión cognitivo-social, la visión de la autorregulación comprende tres fases: auto-observación (auto-monitorización), autojuicio y auto-reacción. Zimmerman citado por De la Fuente Arias, J. (2017), ha desarrollado un modelo cíclico, centrado en las interacciones recíprocas. Incluye tres fases: 1) Planificación, o fase que precede al aprendizaje, centrada en el establecimiento de metas y de la percepción de autoeficacia; 2) Control volitivo durante la ejecución, con implementación de estrategias de control y centrado en la ejecución de la tarea; 3) Auto-reflexión, con autoevaluación de la consecución de los logros. Esta fase tiene efectos cognitivos, conductuales y motivacionales. Las habilidades de autorregulación pueden aprenderse, siguiendo cada una de las fases del modelo.

El pensamiento crítico autorregulado es considerado como "monitoreo auto consciente de las actividades cognitivas propias, de los elementos utilizados en esas actividades, y de los resultados obtenidos, aplicando particularmente habilidades de análisis y de evaluación a los juicios inferenciales propios, con la idea de cuestionar, confirmar, validar, o corregir el razonamiento o los resultados propios".

Las dos sub habilidades, en este caso, son el auto examen y el auto corrección. ¿Ejemplos? Fácil - examinar sus puntos de vista sobre un asunto controversial siendo sensible a las posibles influencias de sus predisposiciones personales o de su propio interés; al escuchar a una persona, cuestionarse mientras ella habla para asegurarse de que realmente está entendiendo lo que ella está diciendo sin introducir sus propias ideas; monitorear qué tan bien parece estar comprendiendo lo que está leyendo o experimentando; acordarse de diferenciar sus opiniones y presunciones personales de las del autor de un pasaje o texto; asegurarse recalculando nuevamente las cifras; variar su velocidad y método de lectura de acuerdo con el tipo de material y el propósito de esta; reconsiderar su interpretación o juicio en busca de realizar un análisis más profundo de los hechos del caso; revisar sus respuestas en base a los errores que descubrió en su trabajo; cambiar su conclusión al darse cuenta de que ha juzgado erróneamente la importancia de ciertos factores en su decisión inicial. 


\section{Metodología}

La investigación se sustenta en el método hipotético deductivo, enfoque cuantitativo, respaldado en "la recolección de datos para probar hipótesis, con base en la medición numérica y el análisis estadístico, para establecer patrones de comportamiento y probar teorías" (Hernández, Fernández y Baptista, 2014, p. 4), diseño cuasi experimental, la cual se manipulan deliberadamente, al menos una variable independiente para observar su efecto y relación con una o más variables dependientes, sólo que difieren de los experimentos "puros" en el grado de seguridad o confiabilidad que pueda tenerse sobre la equivalencia inicial de los grupos. En este caso el diseño es con dos grupos uno experimental y otro de control, con pre prueba- post prueba y grupos intactos (uno de ellos de control).

La población del presente estudio estuvo constituida por 420 estudiantes del I ciclo de la universidad nacional de San Martín - Tarapoto, con un muestreo no probabilístico, se encuestaron a 180 estudiantes. Para la presente investigación se utilizó la técnica de la encuesta, acompañado de un cuestionario como instrumento, que sirvió para el recojo de información.

\section{Resultados}

Tabla 1

Estadísticas de muestras relacionadas (Habilidades del pensamiento crítico)

\begin{tabular}{cccccc}
\hline & & Media & N & $\begin{array}{c}\text { Desviación } \\
\text { estándar }\end{array}$ & $\begin{array}{c}\text { Media de error } \\
\text { estándar }\end{array}$ \\
\hline $\begin{array}{c}\text { Grupo } \\
\text { Experimental. }\end{array}$ & Pre & 75,70 & 180 & 7,806 & 0,582 \\
\hline \multirow{2}{*}{ Grupo Control } & Pre & 765,36 & 180 & 5,911 & 0,441 \\
& Pos & 81,07 & 180 & 8,534 & 0,636 \\
\end{tabular}

Fuente: Elaboración propia en base a pre y post test aplicado al grupo experimental y grupo control

Se observa los resultados del grupo experimental, en el pre test se tiene un promedio de 75,70 puntos quienes fueron sometidos en el experimento quienes llegaron a tener el promedio de 105,36 con una diferencia del 29,66 punto, que representa el 39,18\% de incremento con respecto al pre test. Mientras que en el grupo control en el pre test llegaron a tener 76,08 puntos y que al evaluarlo en la prueba de salida alcanzaron los puntajes del 81,07 que representa un incremento de 4,99 que representa al 6,56\%. Por otro lado, se evidencia que ambos grupos provienen de una población homogénea. 
Tabla 2

Resultados del pre test - pos tes en relación a las habilidades del pensamiento critico

\section{Diferencias relacionadas}

\begin{tabular}{|c|c|c|c|c|c|c|c|c|}
\hline \multirow[t]{2}{*}{ Grupo } & \multirow[t]{2}{*}{ Media } & \multirow[t]{2}{*}{$\begin{array}{l}\text { Desviación } \\
\text { estándar }\end{array}$} & \multirow[t]{2}{*}{$\begin{array}{c}\text { Media del } \\
\text { error } \\
\text { estándar }\end{array}$} & \multicolumn{2}{|c|}{$\begin{array}{c}95 \% \text { de intervalo de } \\
\text { confianza de la } \\
\text { diferencia }\end{array}$} & \multirow[t]{2}{*}{$\mathrm{t}$} & \multirow[t]{2}{*}{$\mathrm{gl}$} & \multirow[t]{2}{*}{$\begin{array}{c}\text { Sig. } \\
\text { (bilateral) }\end{array}$} \\
\hline & & & & Inferior & Superior & & & \\
\hline Experimental & 29,666 & 5,667 & 0,422 & 28,822 & 30,489 & 70,208 & 179 & 0,000 \\
\hline Control & 4,992 & 3,584 & 0,267 & 0,022 & 1,033 & 1,893 & 179 & 0,068 \\
\hline
\end{tabular}

Fuente: Elaboración propia en base a pre y post test aplicado al grupo experimental y grupo control

Se observa que hay evidencia para rechazar la hipótesis nula, por tanto los puntajes son diferentes, los puntajes son mayores en el pos test comparado con el pre test, donde: $\mathrm{P}$ sig $<0,05$ - $0,000<0,05$. En este caso se rechaza la hipótesis nula y se acepta la hipótesis alternativa donde la efectividad de la pedagogía universitaria basada en competencias genéricas en el desarrollo de habilidades del pensamiento crítico en los estudiantes del I ciclo de la Universidad Nacional de San Martín, 2019; es decir los puntajes del pos test son altos.

Tabla 3.

Estadísticas de muestras relacionadas (Dimensión: inferencial)

\begin{tabular}{cccccc}
\hline & Media & N & $\begin{array}{c}\text { Desviación } \\
\text { estándar }\end{array}$ & $\begin{array}{c}\text { Media de error } \\
\text { estándar }\end{array}$ \\
\hline $\begin{array}{c}\text { Grupo } \\
\text { Experimental. }\end{array}$ & Pre & 25,16 & 180 & 4,922 & 0,367 \\
\hline \multirow{2}{*}{ Prupo Control } & 35,68 & 180 & 3,268 & 0,244 \\
& Pre & 25.50 & 180 & 4,195 & 0,313 \\
& Pos & 28,16 & 180 & 4,052 & 0,302
\end{tabular}
control

Fuente: Elaboración propia en base a pre y post test aplicado al grupo experimental y grupo

Se observa los resultados del grupo experimental, según la dimensión inferencial en el pre test se tiene un promedio de 25,16 puntos quienes fueron sometidos en el experimento quienes llegaron a tener el promedio de 35,68 con una diferencia de 10,52 puntos, que representa el $4181 \%$ de incremento con respecto al pre test. Mientras que en el grupo control en el pre test llegaron a tener 25,50 puntos y que al evaluarlo en la prueba de salida alcanzaron los puntajes del 28,16 que representa un incremento del 2,66 punto equivalente a 10,43\%. Además las muestras obtenidas provienen de una población homogénea. 


\section{Tabla 4}

Resultados del pre test - pos tes en relación a las habilidades del pensamiento crítico, dimensión: inferencial

\section{Diferencias relacionadas}

\begin{tabular}{|c|c|c|c|c|c|c|c|c|}
\hline \multirow{3}{*}{ Grupo } & & \multirow{3}{*}{$\mathrm{t}$} & \multirow{3}{*}{$\mathrm{gl}$} & \multirow{3}{*}{$\begin{array}{c}\text { Sig. } \\
\text { (bilateral) }\end{array}$} \\
\hline & \multirow[t]{2}{*}{ Media } & \multirow[t]{2}{*}{$\begin{array}{c}\text { Desviación } \\
\text { estándar }\end{array}$} & \multirow{2}{*}{$\begin{array}{l}\text { Media del } \\
\text { error } \\
\text { estándar }\end{array}$} & \multicolumn{2}{|c|}{$\begin{array}{l}95 \% \text { de intervalo de } \\
\text { confianza de la } \\
\text { diferencia }\end{array}$} & & & \\
\hline & & & & Inferior & Superior & & & \\
\hline Experimental & 10,522 & 3,458 & 0,258 & 10,014 & 11,031 & 40,820 & 179 & 0,000 \\
\hline Control & 2,662 & 2,479 & 0,185 & 0,192 & 0,537 & 0,932 & 179 & 0,352 \\
\hline
\end{tabular}

Fuente: Elaboración propia en base a pre y post test aplicado al grupo experimental y grupo control

Se observa que hay evidencia para rechazar la hipótesis nula, por tanto los puntajes son diferentes, los puntajes son mayores en el pos test comparado con el pre test, donde: $\mathrm{P}$ sig $<0,05$ - $0,000<0,05$. Entonces se rechaza la hipótesis nula y se acepta la hipótesis alternativa donde la pedagogía universitaria basada en competencias genéricas en el desarrollo de habilidades del pensamiento crítico según dimensión inferencial resultó efectiva en los estudiantes del I ciclo de la Universidad Nacional de San Martín, 2019; es decir los puntajes del pos test son altos, a un nivel de significancia del $5 \%$, con una p-valor 0.000 , esto nos indica que es significativo.

Tabla 5.

Estadísticas de muestras relacionadas (Dimensión: explicación)

\begin{tabular}{cccccc}
\hline & & Media & N & $\begin{array}{c}\text { Desviación } \\
\text { estándar }\end{array}$ & $\begin{array}{c}\text { Media de error } \\
\text { estándar }\end{array}$ \\
\hline Grupo & Pre & 25,72 & 180 & 4,921 & 0,367 \\
Experimental & Pos & 34,89 & 180 & 3,194 & 0,238 \\
\hline \multirow{2}{*}{ Grupo Control } & Pre & 25,54 & 180 & 4,071 & 0,303 \\
& Pos & 26,74 & 180 & 4,006 & 0,299 \\
\hline
\end{tabular}

Fuente: Elaboración propia en base a pre y post test aplicado al grupo experimental y grupo

control

Se observa los resultados del grupo experimental, según la dimensión explicación en el pre test se tiene un promedio de 25,72 puntos, estos sujetos fueron parte de la investigación donde se desarrollaron los talleres, quienes llegaron a tener el promedio de 34,89 con una diferencia de 9.17 puntos, que representa el $35.65 \%$ de incremento con respecto al pre test. Mientras que en el grupo control en el pre test llegaron a tener 25,54 puntos y que al evaluarlo en la prueba de salida alcanzaron los puntajes del 26,74 que representa un incremento del 1.2 punto equivalente a $4,70 \%$. Las muestras provienen de una población homogénea. 


\section{Tabla 6}

Resultados del pre test - pos tes en relación a las habilidades del pensamiento crítico, dimensión: explicación

\section{Diferencias relacionadas}

\begin{tabular}{|c|c|c|c|c|c|c|c|c|}
\hline \multirow{3}{*}{ Grupo } & & & & & & & & \\
\hline & \multirow[t]{2}{*}{ Media } & \multirow[t]{2}{*}{$\begin{array}{l}\text { Desviación } \\
\text { estándar }\end{array}$} & \multirow[t]{2}{*}{$\begin{array}{c}\text { Media del } \\
\text { error } \\
\text { estándar }\end{array}$} & \multicolumn{2}{|c|}{$\begin{array}{l}95 \% \text { de intervalo de } \\
\text { confianza de la } \\
\text { diferencia }\end{array}$} & \multirow[t]{2}{*}{$\mathrm{t}$} & \multirow[t]{2}{*}{$\mathrm{gl}$} & \multirow[t]{2}{*}{$\begin{array}{c}\text { Sig. } \\
\text { (bilateral) }\end{array}$} \\
\hline & & & & Inferior & Superior & & & \\
\hline Experimental & 9,172 & 3,898 & 0,291 & 8,599 & 9,746 & 31,570 & 179 & 0,000 \\
\hline Control & 1,200 & 2,641 & 0,197 & 0,188 & 0,588 & 1,016 & 179 & 0,311 \\
\hline
\end{tabular}

Fuente: Elaboración propia en base a pre y post test aplicado al grupo experimental y grupo

\section{control}

Se observa que hay evidencia para rechazar la hipótesis nula, por tanto, los puntajes son diferentes, los puntajes son mayores en el pos test comparado con el pre test, donde: $\mathrm{P}$ sig < 0,05 - $0,000<0,05$. Entonces se rechaza la hipótesis nula y se acepta la hipótesis alternativa donde la pedagogía universitaria basada en competencias genéricas en el desarrollo de habilidades del pensamiento crítico según dimensión explicación resultó efectiva en los estudiantes del I ciclo de la Universidad Nacional de San Martín, 2019; es decir los puntajes del pos test son altos, a un nivel de significancia del $5 \%$, con una p-valor 0.000 , esto nos indica que es significativo.

\section{Tabla 7}

Estadísticas de muestras relacionadas (Dimensión: autorregulación)

\begin{tabular}{|c|c|c|c|c|c|}
\hline & & Media & $\mathbf{N}$ & $\begin{array}{l}\text { Desviación } \\
\text { estándar }\end{array}$ & $\begin{array}{l}\text { Media de error } \\
\text { estándar }\end{array}$ \\
\hline Grupo & Pre & 24,83 & 180 & 3,902 & 0,291 \\
\hline Experimental & Pos & 34,79 & 180 & 3,289 & 0,245 \\
\hline \multirow{2}{*}{ Grupo Control } & Pre & 25,04 & 180 & 3,345 & 0,249 \\
\hline & Pos & 26,17 & 180 & 3,515 & 0,262 \\
\hline
\end{tabular}

Fuente: Elaboración propia en base a pre y post test aplicado al grupo experimental y grupo

control

Se observa los resultados del grupo experimental, según la dimensión autorregulación en el pre test se tiene un promedio de 24,83 puntos, estos sujetos fueron parte de la investigación donde se desarrollaron los talleres, quienes llegaron a tener el promedio en el pos test de 34,79 con una diferencia de 9.96 puntos, que representa el 40,11\% de incremento con respecto al pre test. En cambio, en el grupo control en el pre test llegaron a tener 25,04 puntos y que al evaluarlo en la prueba de salida alcanzaron los puntajes del 26,17 que representa un incremento del 1.13 punto equivalente a $4,51 \%$. La población muestral es homogénea. 


\section{Tabla 8}

Resultados del pre test - pos tes en relación a las habilidades del pensamiento critico, dimensión: autorregulación

\begin{tabular}{|c|c|c|c|c|c|c|c|c|}
\hline \multirow{3}{*}{ Grupo } & \multicolumn{5}{|c|}{ Diferencias relacionadas } & \multirow{3}{*}{$\mathrm{t}$} & \multirow{3}{*}{ gl } & \multirow{3}{*}{ Sig. (bilateral) } \\
\hline & \multirow[t]{2}{*}{ Media } & \multirow[t]{2}{*}{$\begin{array}{c}\text { Desviación } \\
\text { estándar }\end{array}$} & \multirow[t]{2}{*}{$\begin{array}{l}\text { Media del } \\
\text { error } \\
\text { estándar }\end{array}$} & \multicolumn{2}{|c|}{$\begin{array}{c}95 \% \text { de intervalo } \\
\text { de confianza de la } \\
\text { diferencia }\end{array}$} & & & \\
\hline & & & & Inferior & Superior & & & \\
\hline & 9,961 & 2,998 & 0,223 & 9,520 & 10,402 & 44,579 & 179 & 0,000 \\
\hline rol & 1,133 & 2,285 & 0,170 & 0,203 & 0,469 & 0,783 & 179 & 0,435 \\
\hline
\end{tabular}

Fuente: Elaboración propia en base a pre y post test aplicado al grupo experimental y grupo control

Se observa que hay evidencia para rechazar la hipótesis nula, por tanto los puntajes son diferentes, los puntajes son mayores en el pos test comparado con el pre test, donde: $\mathrm{P}$ sig $<0,05$ - $0,000<0,05$. Entonces se rechaza la hipótesis nula y se acepta la hipótesis alternativa donde la pedagogía universitaria basada en competencias genéricas en el desarrollo de habilidades del pensamiento crítico según dimensión autorregulación resultó efectiva en los estudiantes del I ciclo de la Universidad Nacional de San Martín, 2019; es decir los puntajes del pos test son altos, a un nivel de significancia del $5 \%$, con una p-valor 0.000 , esto nos indica que es significativo.

\section{Discusión}

En la tabla 1, se observan los resultados del grupo experimental, en el pre test se tiene un promedio de 75,70 puntos quienes fueron sometidos en el experimento quienes llegaron a tener el promedio de 105,36 con una diferencia del 29,66 punto, que representa el 39,18\% de incremento con respecto al pre test. Mientras que en el grupo control en el pre test llegaron a tener 76,08 puntos y que al evaluarlo en la prueba de salida alcanzaron los puntajes del 81,07 que representa un incremento de 4,99 que representa al 6,56\%. Por otro lado, se evidencia que ambos grupos provienen de una población homogénea. Al respecto Biggs (2011), considera que la pedagogía universitaria está orientada asegurar que los docentes logren que los estudiantes construyan conocimientos profundos, significativos, relevantes, que les permitan insertarse adecuadamente en el mundo académico y profesional. Asimismo, Barnett (1999) propone renovar la pedagogía universitaria de forma tal que prepare a los estudiantes para afrontar la supercomplejidad del mundo actual, en el cual nociones fundamentales como verdad y justicia muchas tienen interpretaciones contenciosas entre las cuales es difícil tomar partido.

En la tabla 3, se observan los resultados del grupo experimental, según la dimensión inferencial en el pre test se tiene un promedio de 25,16 puntos quienes fueron sometidos en el experimento quienes llegaron a tener el promedio de 35,68 con una diferencia de 10,52 puntos, que representa el $4181 \%$ de incremento con respecto al pre test. Mientras que en el grupo control en el pre test llegaron a tener 25,50 puntos y que al evaluarlo en la prueba de salida alcanzaron los puntajes del 28,16 que representa un incremento del 2,66 punto equivalente a 10,43\%. En este sentido, Monereo (2001) sostiene que estudiar en la universidad, implicará ser capaces de conocer y tener un repertorio amplio de estrategias y técnicas de estudio, que serán entendidas como "un proceso de toma de decisiones, consciente e intencional, que consiste en seleccionar los conocimientos conceptuales, procedimentales y actitudinales, necesarios para cumplimentar un determinado objetivo, siempre en función de las condiciones de la situación educativa en que se 
produce la acción"; esto quiere decir, que no todo la información se debe estudiar, necesariamente de la misma forma, todo dependerá entonces del tipo de conocimiento y el objetivo que nos propongamos lograr con él.

En la tabla 5, se evidencian los resultados del grupo experimental, según la dimensión explicación en el pre test se tiene un promedio de 25,72 puntos, estos sujetos fueron parte de la investigación donde se desarrollaron los talleres, quienes llegaron a tener el promedio de 34,89 con una diferencia de 9.17 puntos, que representa el $35.65 \%$ de incremento con respecto al pre test. Mientras que en el grupo control en el pre test llegaron a tener 25,54 puntos y que al evaluarlo en la prueba de salida alcanzaron los puntajes del 26,74 que representa un incremento del 1.2 punto equivalente a 4,70\%. Al respecto Thompson (2011), considera que es más efectiva la educación que incorpora formas de crear nuevos productos o hipótesis a partir de información existente y significativa para el estudiante, que el pensamiento crítico debería ser transversal a toda la enseñanza universitaria.

En la tabla 7, se evidencian los resultados del grupo experimental, según la dimensión autorregulación en el pre test se tiene un promedio de 24,83 puntos, estos sujetos fueron parte de la investigación donde se desarrollaron los talleres, quienes llegaron a tener el promedio en el pos test de 34,79 con una diferencia de 9.96 puntos, que representa el 40,11\% de incremento con respecto al pre test. En cambio, en el grupo control en el pre test llegaron a tener 25,04 puntos y que al evaluarlo en la prueba de salida alcanzaron los puntajes del 26,17 que representa un incremento del 1.13 punto equivalente a 4,51\%. Además; Pessoa y Urzêda (2012), expresan que se puede impartir el pensamiento crítico en asignaturas como lengua y que es una forma de motivar al estudiante en el proceso dialógico, ya que ayuda a considerar el lenguaje como una práctica social en la cual se involucra al estudiante para que esté sensibilizado ante la injusticia social. Este hecho puede adecuarse a cualquier asignatura e implica al estudiante y al docente por igual adoptando una enseñanza-aprendizaje crítico.

\section{Referencias}

Álvarez de Zayas, C., \& González, E.U. (1998). La Didáctica: un proceso consciente de enseñanza y aprendizaje. Cintex.

Álvarez de Zayas, C. (1998). Fundamentos teóricos de la dirección del proceso docente educativo en la Educación Superior Cubana. La Habana: MES.

Barnett, R. (1999). Los límites de la competencia. Barcelona: Gedisa.

Bloom, B. S. (1990). Taxonomía de los objetivos de la educación. Buenos Aires: El Ateneo.

Brookfield, S. D. (1987). Developing critical thinkers. San Francisco: JosseyBass.

De la Fuente Arias, J. (2017). Autorregulación y procesos de aprendizaje. Aula Magna

Giancarlo, C. A. y Facione P. A. (2001). A look across four years at the disposition toward critical thinking among undergraduate students. The Journal of General Education, 50, (1), 29-55.

González, P. M. (1993). Epistemología histórica y enseñanza. Ayer (12), 135-181.

Guzmán, S., \& Sánchez, P. (2006) Efectos de un programa de capacitación de profesores en el desarrollo de habilidades de pensamiento crítico en estudiantes universitarios en el Sureste de México. Revista electrónica de Investigación Educativa. (8) 2, https:// www.researchgate.net/publication/290601878

Hernández, R., Fernández, C., \& Baptista, P. (2014). Metodología de la investigación. $6^{\mathrm{a} e d .}$ México: McGraw-Hill.

Hernández, R., Fernández, C., \& Baptista, P. (2003). Metodología de la investigación (3a.ed.). México: Mc Graw HILL. 
Laskey, M. L., \& Gibson, P. W. (1997). College study strategies: Thinking and learning. Needham Heights, MA: Allyn and Bacon.

Monereo, C. y J. Pozo (2001). Decálogo para el futuro. Cuadernos de pedagogía 298.

Nagel, E. (2006). La estructura de la ciencia. ISBN 84-493-1870-X. Barcelona. Paidós.

Navarro, G., Vaccarezza, G., González, M., \& Catalán, R. (2015). Construcción de conocimiento en educación superior: Educación de competencias genéricas en la Universidad de Concepción (Chile). (G. Navarro, Ed.) Concepción: Sello editorial Universidad de Concepción.

Olivares. (2016). Desarrollar el pensamiento crítico: "decidiendo en que creer", en Competencias transversales para una sociedad basada en conocimiento, México, Cengage Learning.

Paul, R., \& Elder, L. (2005). Una guía para los educadores en los estándares de competencia para el pensamiento crítico. Fundación para el Pensamiento Crítico. California, E. U.

Pessoa, R., \& Urzêda Freitas, M.D. (2012). Challenges in Critical language teaching. Tesol quarterly, 46(4), 753-776.

Pineda, R., Gilio, M., Andrade, R., Latapí, P., \& Muriel, V. (2017). Modelo Educativo Universitario: Procesos de reflexión participativa y propuesta para su actualización e implementación. Universidad Autónoma de Querétaro.

Salazar D. (2001). La formación interdisciplinaria del futuro profesor de Biología en la actividad científico - investigativa. Tesis de doctorado en Ciencias Pedagógicas, I. S. Pedagógico "E. J. Varona", La Habana, Cuba.

Thompson, C. (2011). Critical thinking across the curriculum: Process over output. International Journal of Humanities and Social Science, 1(9), 1-7.

Tobón, S.. (2005). Formación basada en competencias. Pensamiento complejo, diseño curricular y didáctica. Bogotá (Colombia). Ecoe Ediciones.

Zabalza, M. (2011). Oncensino universitario. Seu cenario e seus protagonistas. Porto Alegre: Artmed. 